\title{
FXYD2 mRNA expression represents a new independent factor that affects survival of glioma patients and predicts chemosensitivity of patients to temozolomide
}

\author{
Kaijia Zhou ${ }^{1,2}$, Tao Jiang ${ }^{2,3,4,5^{*}}$ (D) Yanwei Liü ${ }^{3}$, Zheng Zhao ${ }^{2,3}$, Lijie Huang ${ }^{2}$ and Guanzhang Li ${ }^{2}$
}

\begin{abstract}
Purpose: Glioma is the most common primary intracranial tumor. Owing to the poor prognosis associated with high-grade gliomas, there is an urgent need to identify biomarkers related to prognosis and treatment sensitivity. Here, we analyze the expression of FXYD2 mRNA in gliomas, and explore its clinical prognostic value and significance in this disease.

Methods: Clinical features, FXYD2 mRNA expression levels, and survival data were analyzed for 516 glioma patients from the Chinese Glioma Genome Map Project, 481 from the cancer genome map datbase and 268 from the molecular braintumor database. The expression patterns for FXYD2 mRNA were compared using the chi-square test, and overall survival (OS) of glioma patients was evaluated according to FXYD2 mRNA expression levels. The factors affecting glioma survival were evaluated by Cox univariate and multivariate regression analysis.

Results: FXYD2 mRNA expression was related to the grade of gliomas. The higher the level, the lower the expression. Meanwhile related to the pathological classification of gliomas. Oligodendroglioma, IDH-mutant and 1p/19q-codeleted was higher than Astrocytoma, IDH-mutant, higher than Glioblastoma, IDH-wildtype. Moreover, temozolomide (TMZ) chemotherapy was found to be an independent factor affecting survival in patients with high FXYD2 mRNA expression, but not in patients with low expression.
\end{abstract}

Conclusion: FXYD2 mRNA expression represents a new independent factor affecting the survival of glioma patients and may serve as an independent prognostic indicator to predict the sensitivity of gliomas to TMZ.

Keywords: FXYD2, Glioma, Chemotherapy, Prognosis, Molecular profile

\section{Introduction}

Gliomas, the most common primary malignant tumor of the brain [1], was classified in 2021 by the World Health Organization (WHO) based on histopathology, isocitrate dehydrogenase (IDH) mutation and $1 \mathrm{p} 19 \mathrm{q}$

*Correspondence: taojiang1964@163.com

${ }^{2}$ Beijing Neurosurgical Institute, Capital Medical University,

Beijing 100070, China

Full list of author information is available at the end of the article co-deletion [2]. Moreover, the prognosis of high-grade gliomas remains poor, particularly in patients with glioblastoma who have a 5-year survival rate of only $5 \%$ [1, 3], even after administration of the standard three treatments: maximum surgical resection, radiotherapy, and chemotherapy. In fact, the average survival time for glioblastoma patients is only 14 months [4]. Due to the heterogeneity of gliomas [5] and different drug resistance to temozolomide (TMZ) [6-9], the prognosis of different glioma patients is quite different. Meanwhile, the 
application of high-throughput technology for the molecular classification of gliomas as well as for screening differentially expressed genes and drug resistance genes has become a research hotspot to facilitate the development of corresponding targeted drugs.

$\mathrm{Na}$ /K-ATPase is an oligomeric transmembrane protein composed of $\alpha, \beta$, and $\gamma$ subunits that functions to maintain the dynamic membrane potential and is associated with many cellular functions as well as the pathogenesis of specific diseases [10]. Specifically, $\mathrm{Na} / \mathrm{K}$-ATPase upregulation has been reported in various cancers [11-15]. Meanwhile, inhibiting $\mathrm{Na} / \mathrm{K}-\mathrm{ATPase}$ activation and expression effectively inhibits cancer cell proliferation and survival [16, 17]. FXYD2 (sodium/ potassium-transporting ATPase subunit gamma) is the $\gamma$ subunit of the $\mathrm{Na} / \mathrm{K}$-ATP enzyme and functions as a regulator of the enzyme activity [18]. Interestingly, a previous study reported that in ovarian clear cell carcinoma (CCC) patients, the expression level of FXYD2 was positively correlated with patient prognosis. Specifically, upregulated FXYD2 expression increased the sensitivity of ovarian CCC cells to the $\mathrm{Na} / \mathrm{K}$-ATPase inhibitor cardiotonic glycoside, thereby enhancing its therapeutic effect. However, the expression pattern and clinical significance of FXYD2 have not yet been reported in gliomas.

Here, through transcriptome sequencing, this study sought to establish the relationship between FXYD2 mRNA expression and the clinical features and survival data for glioma cases collected from the Chinese Glioma Genome Map Project as well as TCGA and REMBRANDT databases.

\section{Data and methods}

\section{Data collection}

Data, including clinical information (sex, age), histopathology, WHO grade, molecular markers (IDH mutation, $1 p / 19 q$ deletion), and follow-up information (survival time), of 516 glioma patients were collected from the Chinese glioma Genome Map Project (CGGA). The enrolled patients were treated by craniotomy in Beijing Tiantan Hospital, China Medical University, Beijing sanbo Hospital Affiliated to Capital Medical University, the First Affiliated Hospital of Nanjing Medical University, Harbin Medical University or General Hospital of Tianjin Medical University from 2005 to 2017. Inclusion criteria were: (1) patients diagnosed with supratentorial cerebellar tumor; (2) patients > 18 years old; (3) patients diagnosed with diffuse glioma; (4) patients signed informed consent. Exclusion criteria were: (1) patients suffering other tumors than glioma (e.g. patients suffering from cerebral lymphoma of melanoma metastasis); (2) patients aged younger than 18 years; (3) patients with other malignat diseases or fixed tumors. All specimens were collected under IRB KY2013-017-01 and frozen in liquid nitrogen within $5 \mathrm{~min}$ after resection. All subjects were unanimously diagnosed as supratentorial diffuse gliomas according to the central pathological examination of an independent committee certified neuropathologist. All patients were classified according to the 2021 World Health Organization (WHO) classification of tumors of the central nervous system [2]. The overall survival (OS) rate of clinical end-point events was calculated from the initial pathological diagnosis to death or last follow-up. This study was approved by the Institutional Ethics Committee of Beijing Tiantan Hospital (KY2014-002-02), and complied with the principles of the Helsinki declaration. All patients provided written informed consent.

\section{mRNA sequencing}

\section{mRNA transcriptome sequencing}

According to the manufacturer's instructions, total RNA was extracted with an RNeasy Mini Kit (Qiagen). Pestle and QIAshredder (Qiagen) were used to crush and homogenate the frozen tissue. The RNA integrity was assessed via electrophoresis using the 2100 bioanalyzer (Agilent Technologies), and only high-quality samples with RNA integrity numbers (RIN) $\geq 6.8$ were used to construct the sequencing library. Briefly, $1 \mu \mathrm{g}$ of total RNA was used in conjunction with the TruSeq RNA library preparation kit (Illumina). With the exception of SuperScript III reverse transcriptase (Invitrogen) used the synthesis of the first strand of cDNA, all other operations were low-throughput. Following PCR amplification, and purification of the junction fragments, the DNA concentration of the junction was determined by quantitative PCR (biological system 7500) with QP1 5'-AATGATACGGCGACCACC GA-3' primers and QP2 5'-CAAGCAGAAGACGGC ATACGAGA- $3^{\prime}$ primers. The length of the DNA fragment was measured using a 2100 bioanalyzer, and the median size of the inserted fragment was $200 \mathrm{bp}$. The RNA-seq library was sequenced using the Illumina HiSeq 2000 Universe 2500 Universe 4000 sequencing system. The library adopts a paired end strategy, with reading lengths of $101 \mathrm{bp}, 125 \mathrm{bp}$, or $150 \mathrm{bp}$. Base invocation was performed using the Illumina Casava v1.8.2 pipeline.

\section{Mapping and quantification}

STAR (v2.5.2b, Dobin et al., 2012) and RSEM (v1.2.31, Li et al., 2011) software were used for RNA-seq mapping and quantification. These reads were then compared with the Human Genome reference (GENCODE v19, hg19) for STAR, after which RSEM was used to calculate the 
sequencing reads for each GENCODE gene. The expression levels of different samples were combined into an FPKM matrix (fragments per million fragments per kilobase transcriptome). Only when the expression level was $>0$ in half the samples was a gene defined as expressed. Finally, we retained only the expressed genes in the mRNA expression profile.

\section{RNA-Seq comparison workflow}

STAR (v2.5.2b) was used to compare the mRNA profiles. For each RNA-seq sample, STAR compares each read group with the human reference genome (GENCODE v19, hg19), and then merges the alignment results.
This workflow generates a BAM file that contains both aligned and unaligned reads (against data). All experimental methods were carried out in accordance with the relevant guidelines and regulations that were previously reported [19]. Preparation, sequencing, and data analysis of the RNA-seq library were the same as that described previously [20].

\section{Identification of IDH mutation and 1p/19q co-deletion}

Aligned the RNA-seq sequencing reads to the human reference genome. and counted the reads position which supporting mutation at chr15:90631838 chr2:209113112 and chr2:209113113 to determinate

Table 1 Relationship between FXYD2 mRNA expression and clinical features in 516 glioma patients

\begin{tabular}{|c|c|c|c|c|c|c|c|c|}
\hline \multirow[t]{2}{*}{ Parameter } & \multirow[t]{2}{*}{ Variable } & \multirow[t]{2}{*}{$\mathbf{N}$} & \multicolumn{4}{|c|}{ FXYD2 mRNA expression } & \multirow[t]{2}{*}{$x^{2}$} & \multirow[t]{2}{*}{$P$ value } \\
\hline & & & Low & $\%$ & High & $\%$ & & \\
\hline \multirow[t]{2}{*}{ Sex } & Female & 225 & 115 & 51.1 & 110 & 48.9 & 0.197 & 0.657 \\
\hline & Male & 291 & 143 & 49.1 & 148 & 50.9 & & \\
\hline \multirow[t]{2}{*}{$\operatorname{Age}^{a}$} & $<43$ & 238 & 119 & 50.0 & 119 & 50.0 & 0.000 & 1.000 \\
\hline & $\geq 43$ & 278 & 139 & 50.0 & 139 & 50.0 & & \\
\hline \multirow[t]{2}{*}{ Progression status } & Primary & 301 & 129 & 42.9 & 172 & 57.1 & 14.743 & 0.000 \\
\hline & Recurrent & 215 & 129 & 60.0 & 86 & 40.0 & & \\
\hline \multirow[t]{3}{*}{ Histopathological types $(\mathrm{HE})^{\mathrm{b}}$} & oligodendroglioma & 114 & 31 & 27.2 & 83 & 72.8 & 31.937 & 0.000 \\
\hline & astrocytoma & 218 & 117 & 53.7 & 101 & 46.3 & & \\
\hline & glioblastoma & 184 & 110 & 59.8 & 74 & 40.2 & & \\
\hline \multirow[t]{2}{*}{ IDH mutation status } & Wildtype & 224 & 155 & 69.2 & 69 & 30.8 & 58.347 & 0.000 \\
\hline & Mutant & 292 & 103 & 35.3 & 189 & 64.7 & & \\
\hline \multirow[t]{2}{*}{$1 p / 19 q$ codeletion status } & Non-codel & 401 & 231 & 57.6 & 170 & 42.4 & 41.636 & 0.000 \\
\hline & Codel & 115 & 27 & 23.5 & 88 & 76.5 & & \\
\hline \multirow[t]{3}{*}{ MGMT methylation_status } & methylated & 244 & 115 & 47.1 & 129 & 52.9 & 0.564 & 0.453 \\
\hline & un-methylated & 169 & 86 & 50.9 & 83 & 49.1 & & \\
\hline & NA & 103 & 57 & 55.3 & 46 & 44.7 & & \\
\hline \multirow[t]{4}{*}{2021 WHO classification } & $\begin{array}{l}\text { Oligodendroglioma, IDH-mutant and } \\
1 \mathrm{p} / 19 \mathrm{q} \text {-codeleted }\end{array}$ & 114 & 31 & 27.2 & 83 & 72.8 & 64.092 & 0.000 \\
\hline & Astrocytoma, IDH-mutant & 186 & 77 & 41.4 & 109 & 58.6 & & \\
\hline & Glioblastoma, IDH-wildtype. & 147 & 97 & 66.0 & 50 & 34.0 & & \\
\hline & Astrocytoma, IDH-wildtype $(\mathrm{NEC})^{c}$ & 69 & 53 & 76.8 & 16 & 23.2 & & \\
\hline \multirow[t]{3}{*}{2021 WHO grade } & 2 & 134 & 60 & 44.8 & 74 & 55.2 & 10.951 & 0.004 \\
\hline & 3 & 198 & 88 & 44.4 & 110 & 55.6 & & \\
\hline & 4 & 184 & 110 & 59.8 & 74 & 40.2 & & \\
\hline \multirow[t]{3}{*}{ Complete excision } & No & 126 & 61 & 48.4 & 65 & 51.6 & 0.095 & 0.758 \\
\hline & Yes & 372 & 186 & 50.0 & 186 & 50.0 & & \\
\hline & NA & 18 & 11 & 61.1 & 7 & 38.9 & & \\
\hline \multirow[t]{2}{*}{ Radiotherapy } & No & 108 & 54 & 50.0 & 54 & 50.0 & 0.000 & 1.000 \\
\hline & Yes & 408 & 204 & 50.0 & 204 & 50.0 & & \\
\hline \multirow[t]{2}{*}{ Chemotherapy } & No & 124 & 60 & 48.4 & 64 & 51.6 & 0.170 & 0.680 \\
\hline & Yes & 392 & 198 & 50.5 & 194 & 49.5 & & \\
\hline
\end{tabular}

a The patients were divided into two groups according to the median age of 43 years

${ }^{b}$ The histological and pathological types were determined based exclusively on morphological features on hematoxylin-eosin sections

${ }^{\mathrm{c}} \mathrm{NEC}$ means that the necessary molecular markers have been successfully detected, but the results cannot be classified into the current who glioma classification 
the IDH mutation status. Gene expression was used to predict $1 \mathrm{p} / 19 \mathrm{q}$ status. Firstly, obtained the proportional gene expression profiles of $1 p$ and $19 q$ genes sorted by genomic location. Then smoothed $1 \mathrm{p}$ and $19 \mathrm{q}$ expression levels using a sliding 100 gene window. Finally, used the clustering method to determine the co-deletion status of $1 \mathrm{p}$ and $19 \mathrm{q}$.

\section{O6-methylguanine-DNA methyltransferase (MGMT) methylation detection}

After the samples were treated with bisulfite, the promoter region was sequenced to identify the methylation sites, and the average methylation ratio of each site was calculated. According to the research results, the cut-off value of the average methylation level in the sequencing section is set to $8 \%$ : the samples with the average methylation level $\leq 8 \%$ are MGMT methylation negative, and the samples with the average methylation level $>8 \%$ are MGMT methylation positive.

\section{Histological types}

In this study, "Histopathological types (HE)" in Table.1 means the histological and pathological types were determined based exclusively on morphological features on hematoxylin-eosin sections. While "histological types" in "2021 WHO classification" Table.1 were based on "integrated" histological and molecular features.

\section{Verification group data collection}

The clinical, histopathological, and survival follow-up data, as well as FXYD2 mRNA sequencing data, for glioma patients were collected from two open independent datasets. Among them, 481 cases were from the cancer genome map database (TCGA, http://www.cgga.org.cn/ download_other.jsp), and 268 were from the molecular brain tumor database (REMBRANDT, http://www.cgga. org.cn/download_other.jsp).

\section{Statistical analysis}

$\mathrm{R}$ software 3.3.2 and SPSS software 25.0 were used to perform all statistical analyses and to generate box scatter plots and survival curves. The normally distributed data were expressed as mean \pm standard deviation $(x \pm s)$. Student's $t$-tests, one-way ANOVA, and LSD- $t$ pairwise comparisons were used to compare FXYD2 mRNA expression in different groups. Kaplan-Meier curve and log rank test were used to analyze the OS of patients in different groups. Univariate and multivariate Cox regression analyses were used to analyze the factors affecting the survival time of glioma patients. All statistical analyses were bilateral, and results were considered statistically significant at $P<0.05$.

\section{Results}

Clinical features and FXYD2 mRNA expression in 516 glioma patients in CGGA

Clinical features included sex, age, recurrence status, histopathology, WHO grade, IDH mutation status, 1p/19q co-deletion status, methylation of O6 methylguanine DNA methyltransferase (MGMT), radiotherapy history, chemotherapy, and integrated diagnosis according to 2021 WHO classification. The histopathology (HE) were determined based exclusively on morphological features on hematoxylin-eosin sections. The 516 glioma patients were then classified as having low or high FXYD2 mRNA expression based on median expression values (Table 1 ). The median expression value was $\geq$ the median expression value and $<$ median expression value (Table 1).

\section{Relationship between FXYD2 mRNA expression and clinical features in glioma patients}

The relationship between clinical characteristics and FXYD2 mRNA expression was analyzed. FXYD2 mRNA expression was not associated with sex $(P=0.657)$, age $(P=1.000)$, methylation of MGMT $(P=0.453)$ or radiotherapy or chemotherapy history $(P=1.000, P=0.680$, respectively) in glioma patients. It was, however, significantly correlated with recurrence $(P=0.000)$, histopathological types (HE) $(P=0.000), 2021$ WHO grade $(P=0.004), I D H$ mutation $(P=0.000), 1 p / 19 q$ co-deletion $(P=0.000)$, and 2021 WHO classification $(P=0.000)$ (Table 1).

\section{FXYD2 MRNA expression is higher in glioma patients with better prognosis}

FXYD2 mRNA expression in glioma patients with different clinical and molecular pathological features was compared using a scatter plot. The clinical features assessed included sex, age, recurrence (Fig. 1), histopathology (HE), WHO grade (Fig. 2), IDH mutation, $1 p / 19 q$ co-deletion status, and 2021 WHO classification (Fig. 3). The results showed that the expression of FXYD2 mRNA was higher in patients with a good prognosis, including those with primary glioma $(P=0.00031)$, oligodendroglioma $(P=5.6 \mathrm{e}-10)$, lower WHO grade $(P=0.00011)$, IDH mutation $(P=2.5 \mathrm{e}-$ $18), \quad 1 p / 19 q$ co-deletion $(P=5.3 \mathrm{e}-12)$, and Oligodendroglioma, IDH-mutant and $1 \mathrm{p} / 19 \mathrm{q}$-codeleted $(P=2.3 \mathrm{e}-20)$.

Moreover, the survival time for glioma patients with high FXYD2 mRNA expression was longer. Kaplan-Meier survival curves were used to explore the effect of FXYD2 mRNA expression on the total survival time of glioma patients. The results show that 


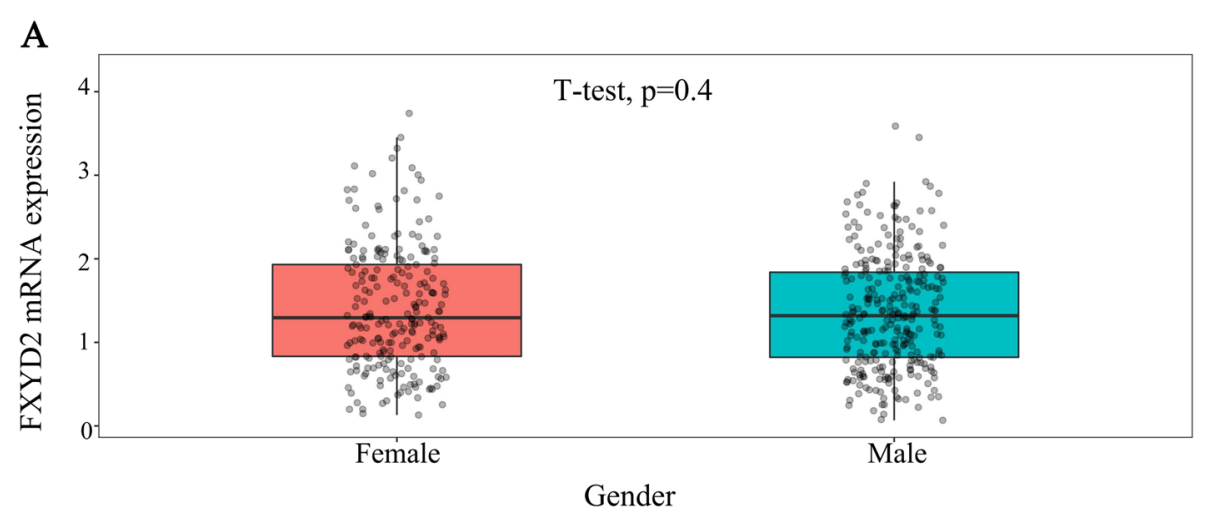

B

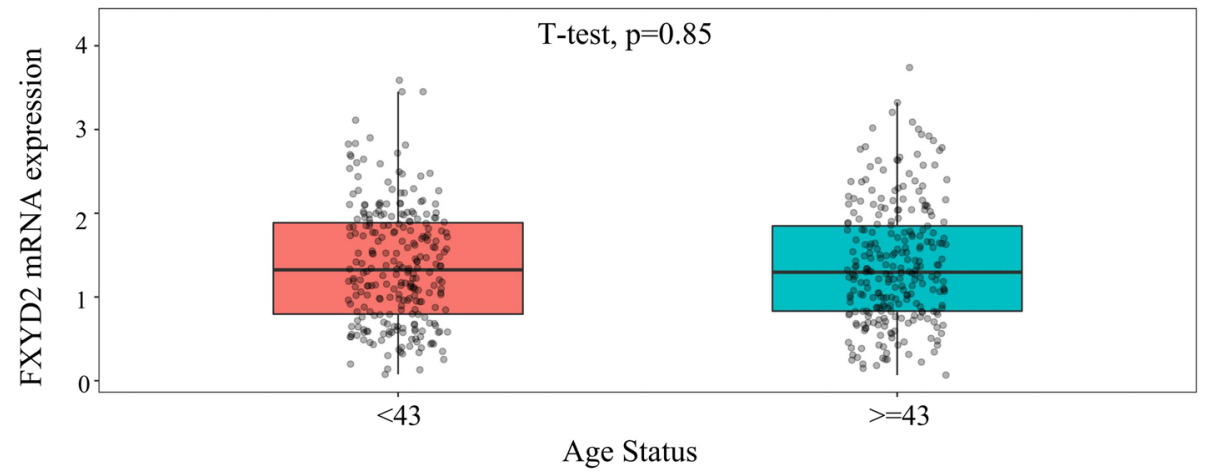

$\mathrm{C}$

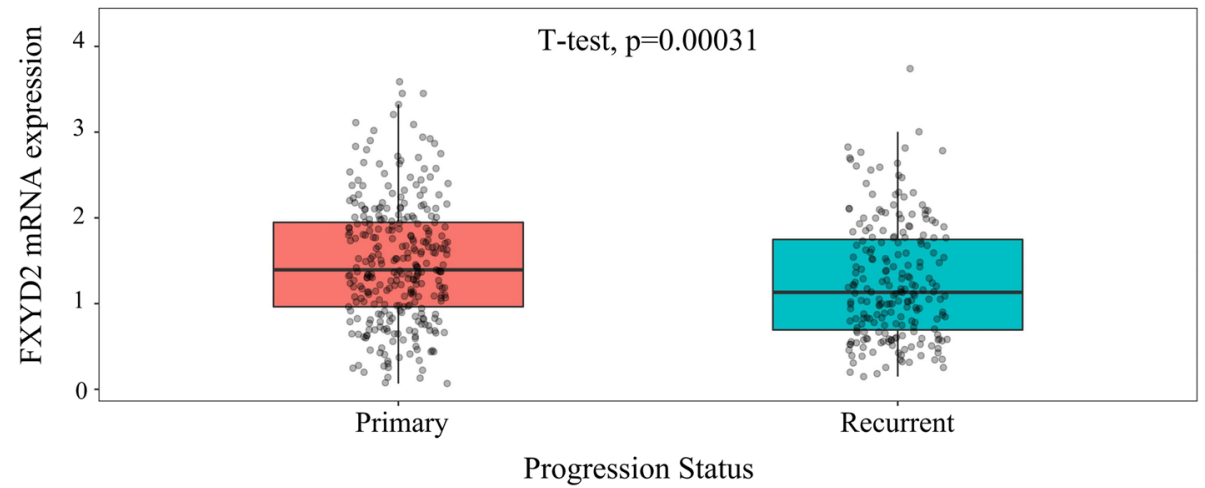

Fig. 1 The expression of FXYD2 mRNA in different gender, age and recurrence status of gliomas in CGGA. The expression of FXYD2 mRNA in 516 glioma patients with different gender $(\mathbf{A})$, age $(\mathbf{B})$ and recurrence status $(\mathbf{C})$ was compared by student's t-test. Data represent mean \pm SD

the survival time of patients with high expression of FXYD2 mRNA was longer than that of patients with low expression of WHOII ( $P=0.000$; Fig. 4A). After stratifying the data according to WHO grade, the same result was observed in patients with all grades of glioma: WHOII $(P=0.011$; Fig. 4B), WHOIII $(P=0.000$; Fig. $4 \mathrm{C})$, and WHOIV $(P=0.043$; Fig. $4 \mathrm{D})$. The same results were also obtained for patients with primary initial gliomas $(P=0.000$; Fig. $4 \mathrm{E})$ and relapse $(P=0.000$; Fig. 4F).
FXYD2 mRNA expression can predict the survival and prognosis of glioma patients

Subgroup analysis showed that different subgroups of glioma patients with high FXYD2 mRNA expression also had longer OS. Among them, low-grade glioma $(P=0.011)$, high-grade glioma $(P=0.000)$, oligodendroglioma $(P=0.004)$, astrocytoma $(P=0.000), I D H$ mutant type $(P=0.000), I D H$ wild type $(P=0.180), 1 p / 19 q$ codeletion type $(P=0.033)$, and $1 p / 19 q$ non-co-deletion type $(P=0.000)$ (Fig. 5). 

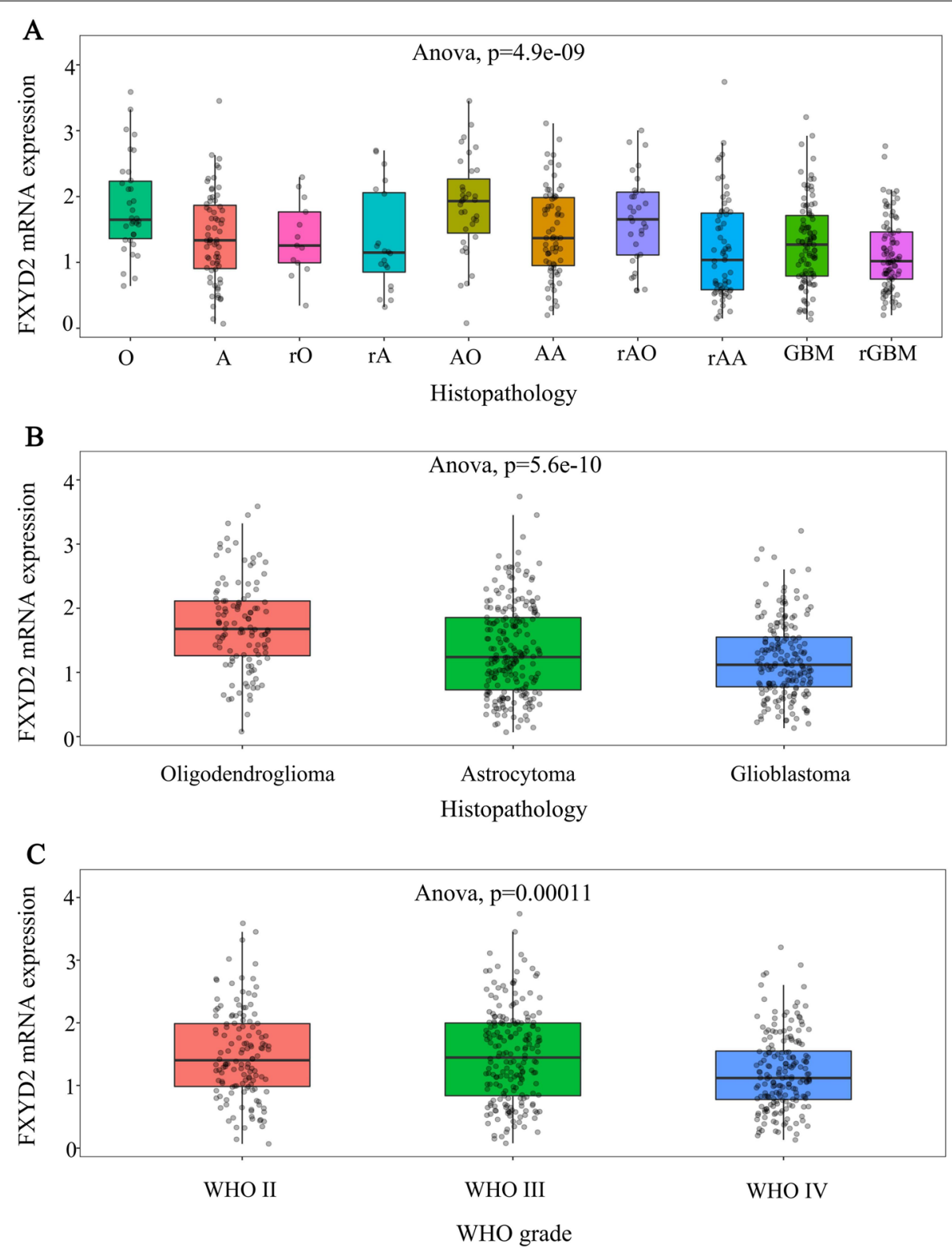

Fig. 2 The expression of FXYD2 mRNA in different histopathology and WHO grade in CGGA. The expression of FXYD2 mRNA in 516 glioma patients with different histopathology $(\mathbf{A}, \mathbf{B})$ and WHO grade $(\mathbf{C})$ was compared by ANOVA. Data represent mean \pm SD

FXYD2 mRNA expression can predict the survival and prognosis of glioma patients in two independent databases

Using Kaplan-Meier survival curves, it was confirmed in two independent databases that glioma patients with high FXYD2 mRNA expression had a longer survival time than patients with low expression from TCGA $(P=0.000$, Fig. 6A) and REMBRANDT database ( $P=0.000$, Fig. 6B). Further subgroup analysis on the two independent datasets showed that patients with low- or high-grade gliomas that had high FXYD2 mRNA expression also exhibited longer survival times (Fig. 6C-F).

\section{FXYD2 mRNA expression is an independent factor affecting the survival of glioma patients}

Univariate Cox analysis was used to identify the factors affecting the survival of glioma patients, including sex, age, recurrence, histopathology (HE), WHO grade, IDH mutation status, $1 p / 19 q$ co-deletion status, methylation status of MGMT, extent of resection, radiotherapy or chemotherapy 

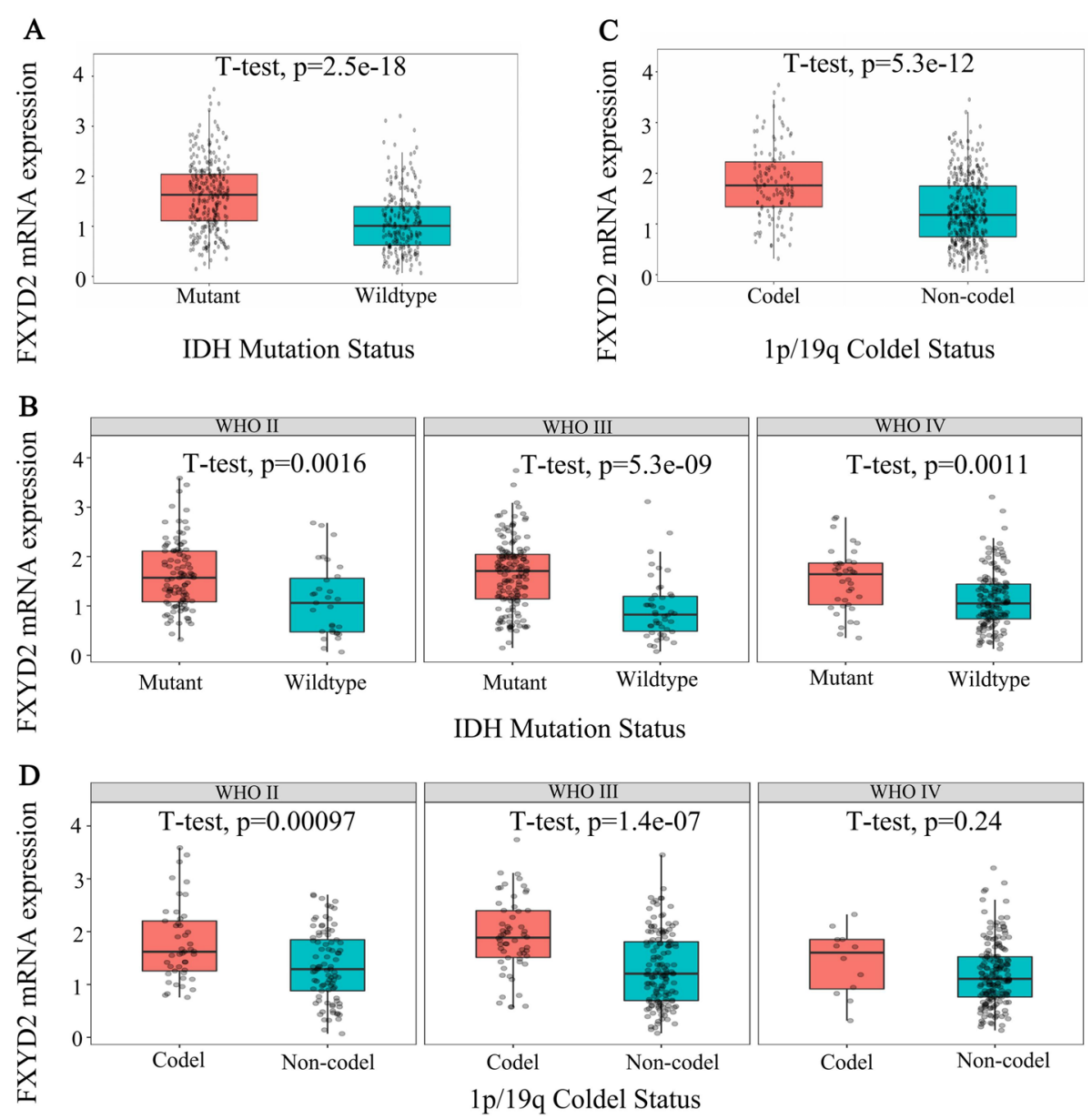

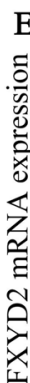

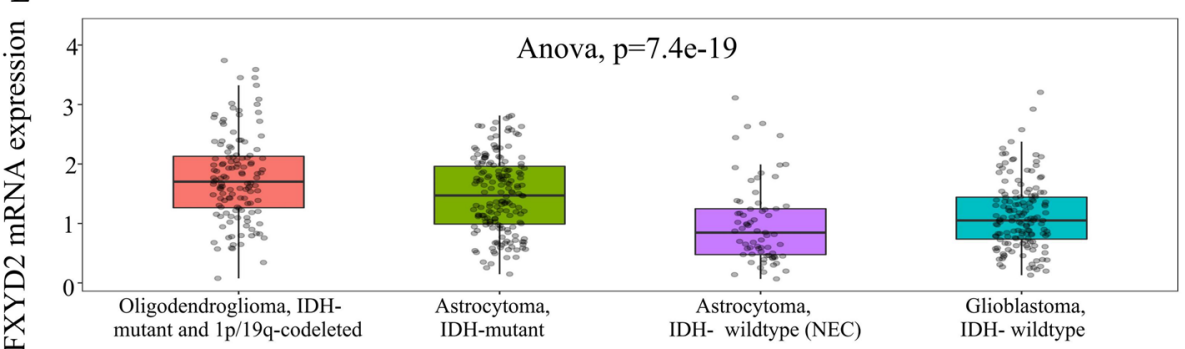

2021 WHO Classification

Fig. 3 The expression of FXYD2 mRNA in different IDH mutation and 1p/19q col-deletion status in CGGA. The expression of FXYD2 mRNA in 516 glioma patients with different IDH mutation status $(\mathbf{A})$ and different $1 \mathrm{p} / 19 \mathrm{q}$ deletion status $(\mathbf{C})$ was compared by student's t-test. The expression of FXYD2 mRNA in patients with different IDH mutation status (B) and 1p/19q deletion status (D) was compared by student's t-test in different WHO grades of glioma patients. There were 134 cases of WHOIl, 198 cases of WHOIII and 184 cases of WHOIV. The expression of FXYD2 mRNA (E) in 516 glioma patients with different WHO classification was compared by ANOVA. Data represent mean \pm SD

status, and FXYD2 mRNA expression. Multivariate Cox analysis showed that high FXYD2 mRNA expression (HR: 0.744, 95\%CI $0.577-0.960, P=0.023$ ), $I D H$ mutation (HR: 0.740, 95\% CI: $0.549-0.997, P=0.048), 1 p / 19 q$ co-deletion (HR: $0.389,95 \%$ CI: $0.260-0.583, P=0.000$ ), and chemotherapy status (HR: $0.607,95 \%$ CI: $0.446-0.825, P=0.001$ ) were independent factors associated with longer patient survival. Meanwhile, age (HR: 1.015, 95\% CI: 1.006-1.024, $P=0.002$ ), relapse (HR: 2.137, 95\% CI: 1.692-2.698, $P=0.000$ ), and WHO grade (HR: $2.700,95 \%$ CI: 1.930 $3.779, P=0.000)$ represented independent factors associated with poor survival (Table 2). 
A

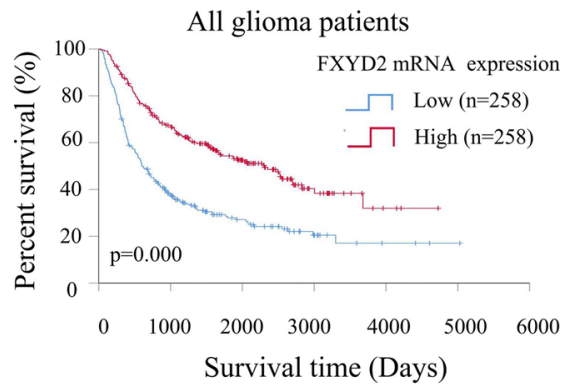

C

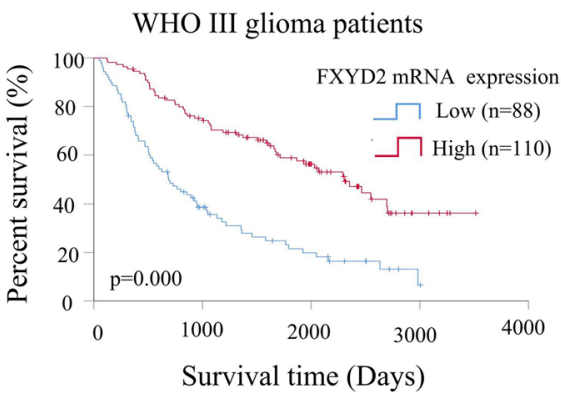

E

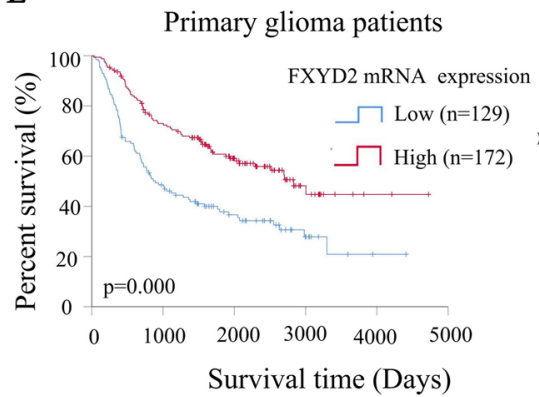

B

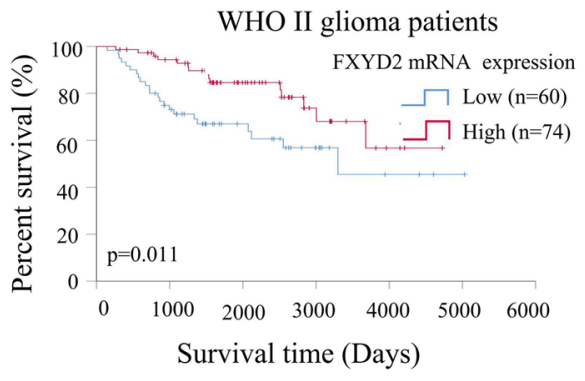

D

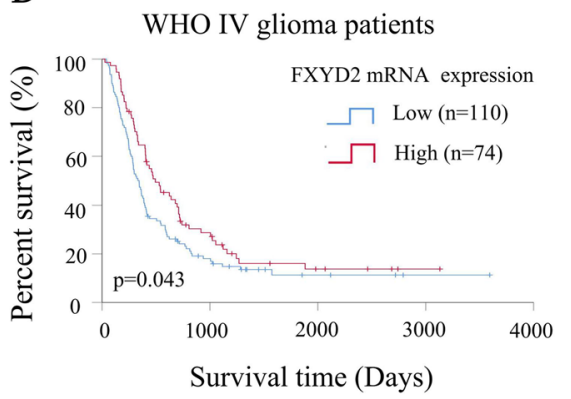

F

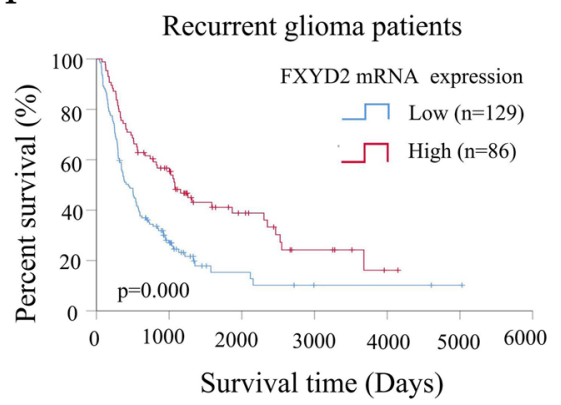

Fig. 4 Kaplan-Meier curves for overall survival (OS) of different FXYD2 mRNA expression in 516 glioma patients with different WHO grades and different recurrent status in CGGA. The Kaplan-Meier curves for overall survival (A-D) of different FXYD2 mRNA expression in glioma patients with different WHO grades. The Kaplan-Meier curves for overall survival (E, F) of FXYD2 mRNA expression in patients with different recurrent gliomas. Log rank test was used to compare the difference between the two survival curves

\section{Increased FXYD2 mRNA expression can predict the chemosensitivity of glioma patients}

According to the median expression of FXYD2 mRNA, the patients were divided into two groups: low or high expression. Univariate Cox analysis was used to investigate the related factors affecting the survival time of glioma patients, including sex, age, recurrence, histopathology, WHO grade, $I D H$ mutation status, $1 p / 19 q$ codeletion status, methylation status of MGMT, extent of resection, radiotherapy, and chemotherapy status. The results of multivariate Cox analysis showed that in the group with high FXYD2 mRNA expression, chemotherapy status (HR: 0.413, 95\% CI: 0.254-0.671, $P=0.000$ ),
IDH mutation status (HR: 0.440, 95\% CI 0.277-0.701, $P=0.001$ ), methylation of MGMT (HR: 0.702, 95\% CI $0.538-0.915, P=0.009$ ) and $1 p / 19 q$ co-deletion (HR: $0.420,95 \%$ confidence interval $0.237-0.747, P=0.003$ ) were independent factors associated with longer survival. Meanwhile, relapse (HR value: 2.940, 95\% CI: 1.9954.332, $P=0.000$ ), WHO grade (HR value: $3.799,95 \% \mathrm{CI}$ : 2.127-6.785, $P=0.000$ ) were independent factors associated with poor survival (Table 3 ). However, in the group with low FXYD2 mRNA expression, chemotherapy status was not an independent factor affecting the survival of patients with glioma (univariate Cox analysis $P=0.132$; multivariate Cox analysis $P=0.174$; Table 3 ). 

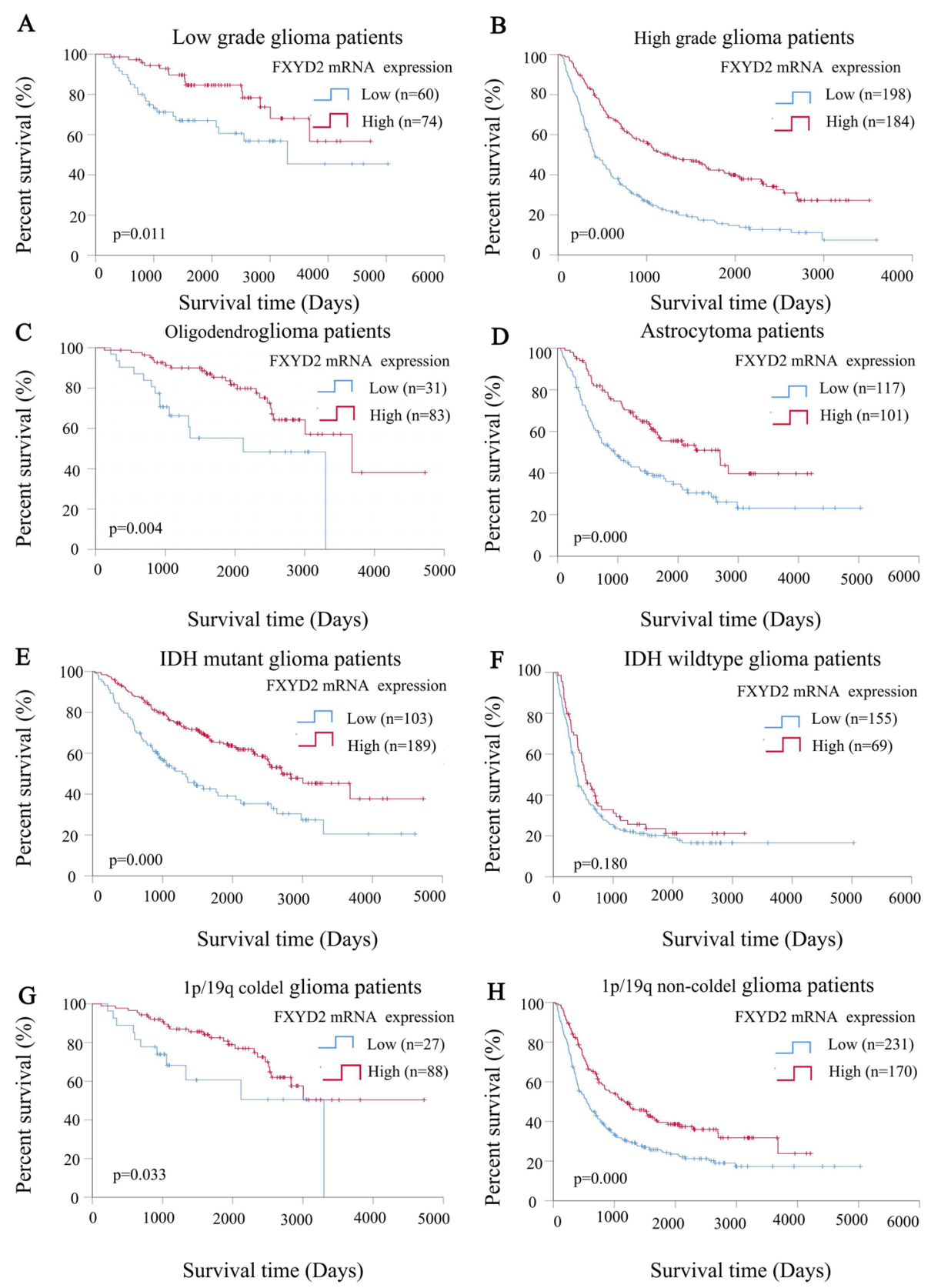

Fig. 5 Kaplan-Meier curves for overall survival of different FXYD2 mRNA expression in CGGA glioma patients with different histologic type, WHO classification, IDH mutation and 1p/19q col-deletion status. The Kaplan-Meier curves for overall survival of different FXYD2 mRNA expression in glioma patients with different WHO grade $(\mathbf{A}, \mathbf{B})$, histologic type $(\mathbf{C}, \mathbf{D})$, IDH mutation $(\mathbf{E}, \mathbf{F})$ and 1p/19q col-deletion status $(\mathbf{G}, \mathbf{H})$. Log rank test was used to compare the difference between the two survival curves

\section{Discussion}

The FXYD2 gene is located on chromosome 11q23 [21], while the FXYD2 protein is the $r$ subunit of the Na-K-ATP enzyme. FXYD2 has been shown to reduce the $\mathrm{Na}$ ion affinity of Na-K-ATP [22], resulting in subsequent inhibition of cell proliferation [23]. However, the expression and application value of FXYD2 mRNA in gliomas have not been previously reported.

This study revealed that the expression of FXYD2 mRNA is related to the degree of malignancy of gliomas. Specifically, higher degree malignancies are associated with lower FXYD2 mRNA expression, suggesting that 

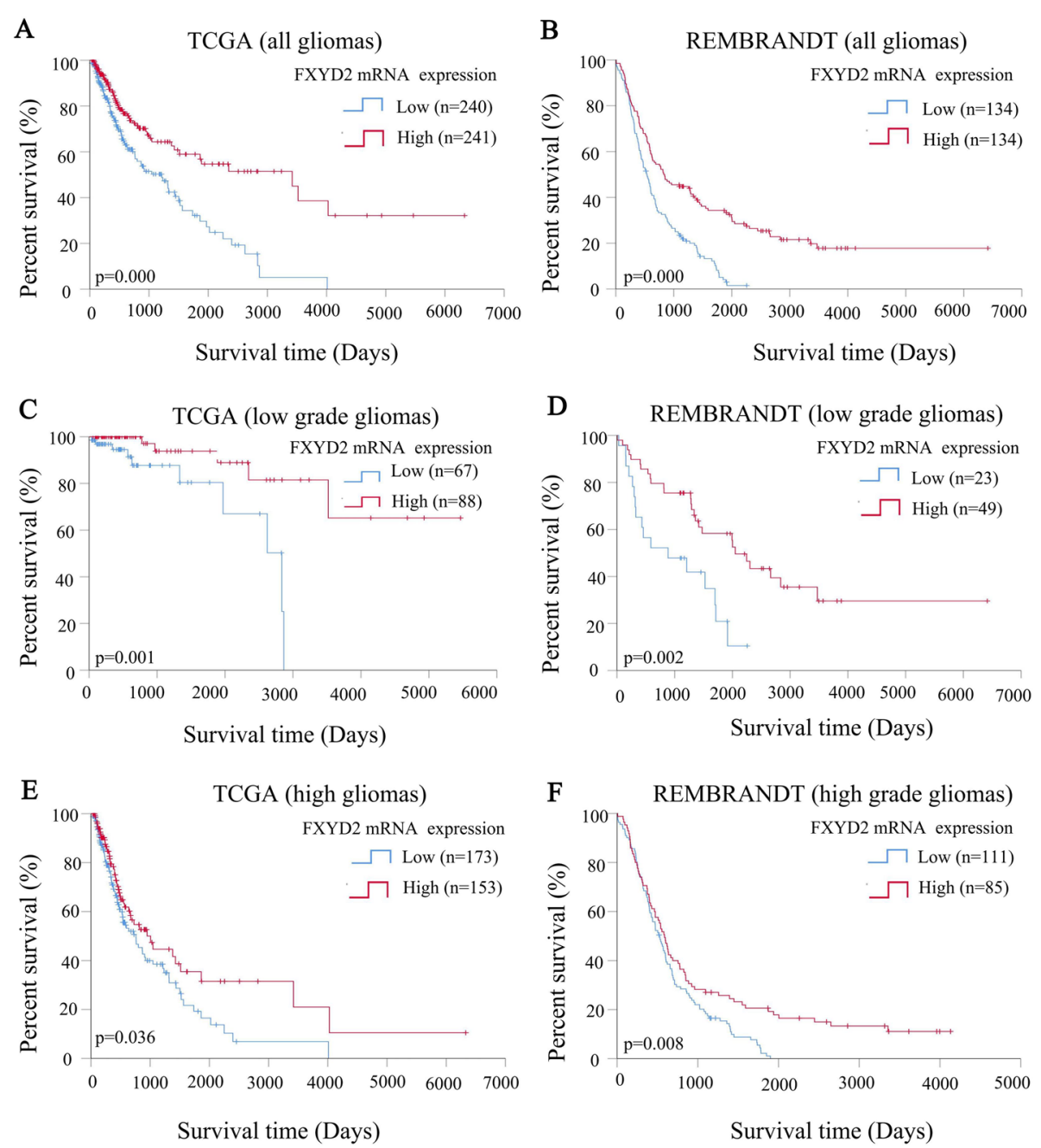

Fig. 6 Kaplan-Meier curves for overall survival of different FXYD2 mRNA expression in TCGA and REMBRANDT databases. The Kaplan-Meier curves for overall survival of different FXYD2 mRNA expression in glioma patients with different WHO grade (A, C, E) in TCGA. The Kaplan-Meier curves for overall survival of different FXYD2 mRNA expression in glioma patients with different WHO grade (B, D, F) in REMBRANDT. Log rank test was used to compare the difference between the two survival curves

FXYD2 mRNA expression can be used as a predictive biomarker for the degree of malignancy of gliomas. Moreover, FXYD2 mRNA expression was found to be related to the survival time of glioma patients with lower expression associated with shorter survival time, suggesting that it can also be used to predict patient survival prognosis. FXYD2 mRNA expression was also related to the chemosensitivity of glioma patients to TMZ. Meanwhile, TMZ represents an independent factor affecting the survival of glioma patients with high expression of FXYD2 mRNA, but not patients with low expression. Hence, we postulate that the expression of FXYD2 mRNA can be used to predict the chemosensitivity to TMZ. Specifically, patients with high FXYD2 mRNA expression will be more likely to respond to TMZ therapy, thereby prolonging survival time, while those with low expression will not benefit from this therapy. These results were similar to those reported by Hsu I-Ling et al. [24] who found that, compared with ovarian cancer cells expressing low levels of FXYD2, those with high expression were more sensitive to cardiosides, while cardiotonic glycosides can effectively inhibit the growth of ovarian cancer cells.

Jin et al. [25] found that FXYD family members are differentially expressed in colon cancer, which is a potential clinical biomarker of colon cancer and participates in the complex biological functions of tumor progression. The expression of FXYD2, FXYD3 and FXYD4 is an independent prognostic factor for for the survival of colon cancer. Currently, the underlying mechanism associated with the effects of FXYD2 in tumors is unclear. The Na-K-ATPase 
Table 2 Correlation analysis between FXYD2 mRNA expression and overall survival among 516 glioma patients in CGGA

\begin{tabular}{|c|c|c|c|c|c|c|}
\hline \multirow[t]{2}{*}{ Parameter } & \multicolumn{3}{|c|}{ Univariate Cox Regression } & \multicolumn{3}{|c|}{ Multivariate Cox Regression } \\
\hline & $P$ value & HR & 95\% Cl (low-up) & $P$ value & HR & $95 \% \mathrm{Cl}$ (low-up) \\
\hline Sex & 0.447 & 1.091 & $0.871-1.366$ & & & \\
\hline Age & 0.000 & 1.027 & $1.017-1.036$ & 0.002 & 1.015 & $1.005-1.024$ \\
\hline Recurrent & 0.000 & 2.117 & $1.690-2.651$ & 0.000 & 2.118 & $1.672-2.684$ \\
\hline Histopathological types (HE) & 0.000 & 1.827 & $1.657-2.015$ & 0.333 & 0.896 & $0.719-1.118$ \\
\hline 2021 WHO glade & 0.000 & 2.804 & $2.377-3.308$ & 0.000 & 2.717 & $1.914-3.856$ \\
\hline IDH mutation status & 0.000 & 0.321 & $0.256-0.403$ & 0.025 & 0.712 & $0.529-0.958$ \\
\hline $1 p / 19 q$ codeletion status & 0.000 & 0.282 & $0.199-0.400$ & 0.000 & 0.403 & $0.265-0.611$ \\
\hline MGMT methylation_status & 0.004 & 0.790 & $0.674-0.926$ & 0.041 & 0.841 & $0.713-0.993$ \\
\hline Complete excision & 0.713 & 1.050 & $0.809-1.364$ & 0.201 & 0.838 & $0.639-1.099$ \\
\hline Radiotherapy & 0.467 & 1.109 & $0.839-1.465$ & 0.244 & 0.825 & $0.598-1.140$ \\
\hline Chemotherapy & 0.230 & 1.176 & $0.903-1.533$ & 0.003 & 0.611 & $0.443-0.841$ \\
\hline FXYD2 mRNA expression & 0.000 & 0.469 & $0.374-0.589$ & 0.018 & 0.959 & $0.927-0.993$ \\
\hline
\end{tabular}

HR Hazard ratio, CI Confidence interval

Table 3 Correlation analysis between chemotherapy and overall survival among glioma patients with high and low FXYD2 mRNA expression in CGGA

\begin{tabular}{|c|c|c|c|c|c|c|}
\hline \multirow[t]{2}{*}{ Parameter } & \multicolumn{3}{|c|}{ Univariate Cox Regression } & \multicolumn{3}{|c|}{ Multivariate Cox Regression } \\
\hline & $P$ value & HR & 95\% Cl (low-up) & $P$ value & HR & 95\% Cl (low-up) \\
\hline \multicolumn{7}{|l|}{ High } \\
\hline Sex & 0.002 & 1.792 & $1.231-2.607$ & 0.123 & 1.396 & $0.914-2.132$ \\
\hline Age & 0.000 & 1.031 & $1.015-1.047$ & 0.137 & 1.012 & $0.996-1.028$ \\
\hline Recurrent & 0.000 & 2.041 & $1.432-2.910$ & 0.000 & 2.951 & $1.977-4.406$ \\
\hline Histopathological types (HE) & 0.000 & 2.037 & $1.754-2.365$ & 0.415 & 0.861 & $0.601-1.233$ \\
\hline 2021 WHO grade & 0.000 & 3.589 & $2.727-4.723$ & 0.000 & 4.037 & $2.208-7.384$ \\
\hline IDH mutation status & 0.000 & 0.261 & $0.181-0.375$ & 0.000 & 0.409 & $0.256-0.655$ \\
\hline $1 p / 19 q$ codeletion status & 0.000 & 0.309 & $0.199-0.480$ & 0.004 & 0.426 & $0.237-0.765$ \\
\hline MGMT methylation_status & 0.021 & 0.729 & $0.558-0.952$ & 0.015 & 0.710 & $0.539-0.935$ \\
\hline Complete excision & 0.669 & 1.092 & $0.730-1.634$ & 0.678 & 0.913 & $0.594-1.403$ \\
\hline Radiotherapy & 0.256 & 1.300 & $0.827-2.043$ & 0.468 & 0.816 & $0.472-1.413$ \\
\hline Chemotherapy & 0.909 & 1.024 & $0.682-1.537$ & 0.001 & 0.427 & $0.259-0.704$ \\
\hline \multicolumn{7}{|l|}{ Low } \\
\hline Sex & 0.101 & 0.786 & $0.589-1.048$ & & & \\
\hline Age & 0.000 & 1.023 & $1.011-1.035$ & 0.024 & 1.014 & $1.002-1.026$ \\
\hline Recurrent & 0.000 & 1.880 & $1.399-2.525$ & 0.000 & 1.859 & $1.360-2.540$ \\
\hline Histopathological types (HE) & 0.000 & 1.583 & $1.387-1.807$ & 0.395 & 0.884 & $0.665-1.175$ \\
\hline 2021 WHO grade & 0.000 & 2.222 & $1.818-2.716$ & 0.000 & 2.416 & $1.564-3.730$ \\
\hline IDH mutation status & 0.000 & 0.472 & $0.347-0.642$ & 0.730 & 0.933 & $0.628-1.385$ \\
\hline $1 p / 19 q$ codeletion status & 0.001 & 0.363 & $0.197-0.668$ & 0.016 & 0.436 & $0.222-0.854$ \\
\hline MGMT methylation_status & 0.036 & 0.813 & $0.669-0.986$ & 0.238 & 0.880 & $0.711-1.088$ \\
\hline Complete excision & 0.805 & 0.958 & $0.679-1.351$ & 0.322 & 0.836 & $0.587-1.191$ \\
\hline Radiotherapy & 0.861 & 0.969 & $0.679-1.382$ & 0.185 & 0.758 & $0.504-1.142$ \\
\hline Chemotherapy & 0.132 & 1.310 & $0.922-1.862$ & 0.279 & 0.788 & $0.512-1.213$ \\
\hline
\end{tabular}

HR Hazard ratio, CI Confidence interval 
serves as the transport system for $\mathrm{Na}$ and $\mathrm{K}$ ions on the cell membrane [26], which serves to maintain the $\mathrm{Na} / \mathrm{K}$ ion concentration gradient inside and outside of the cell. These gradients are essential for maintaining cell volume and membrane potential and also guarantee the maintenance of intracellular homeostasis [26]. They also provide nutrients to the cells and regulate the concentration of intracellular $\mathrm{pH}$ and calcium ions. Meanwhile, increased expression of FXYD2 was found to decrease the activity of $\mathrm{Na} / \mathrm{K}$-ATPase, Moreover, another study has reported that the body senses mechanical pain abnormalities caused by peripheral inflammation through FXYD2 in neurons [27]. Following peripheral tissue inflammation, the interaction between FXYD2 and the $\alpha$ subunit of $\mathrm{Na} / \mathrm{K}$-ATPase is enhanced, causing downregulation of $\mathrm{Na} / \mathrm{K}$-ATPase activity, while increasing neuronal membrane potential depolarization and excitability. The body then senses peripheral inflammatory stimulation signals, resulting in corresponding inflammatory stress and clearance responses.

\section{Conclusions}

This study revealed that the expression of FXYD2 mRNA in gliomas can predict the degree of malignancy and survival time of patients. At the same time, FXYD2 mRNA expression can predict the chemosensitivity of glioma patients to TMZ. However, considering that our study is limited to mRNA, the transcriptional regulation, protein translation, as well as underlying regulatory mechanisms and pathways of FXYD2 remain unclear and require further investigation.

\footnotetext{
Abbreviations

FXYD2: Sodium/potassium-transporting ATPase subunit gamma; OS: Overall survival; WHO: World Health Organization; IDH: Isocitrate dehydrogenase; TMZ: Temozolomide; CCC: Clear cell carcinoma; CGGA: The Chinese glioma Genome Map Project; TCGA: The cancer genome map database; REMBRANDT: The molecular brain tumor database; MGMT: Methylation of 06 methylguanine DNA methyltransferase; HR: Hazard ratio; Cl: Confidence interval; NEC: Not Elsewhere Classified.
}

\section{Acknowledgements}

Not Applicable.

\section{Authors' contributions}

KJZ,TJ and YWL conceived and designed the study. KJZ, LJH and ZZ collected and analyzed the data. KJZ and GZL wrote the first draft of the manuscript. All authors contributed to the manuscript revision, read and approved the final manuscript.

\section{Funding}

This work was supported by the National Natural Science Foundation of China (NSFC)/Research Grants Council (RGC) Joint Research Scheme (81761168038, 2018.01-2021.12) in the design of the study and interpretation of data, and the Beijing Municipal Administration of Hospitals' Mission Plan (SML20180501, 2018.03-2022.02) in the collection and analysis of data.

\section{Availability of data and materials}

The CGGA data used and analyzed during the current study are available from http://www.cgga.org.cn/download.jsp. The TCGA and REMBRANDT datasets used and analysed during the current study are available from http://www. cgga.org.cn/download_other.jsp. Public access to the databases is open.

\section{Declarations}

\section{Ethics approval and consent to participate}

This study was approved by the Institutional Ethics Committee of Beijing Tiantan Hospital (KY2014-002-02); written consent was obtained from all of our enrolled patients.

\section{Consent for publication}

Not Applicable.

\section{Competing interests}

The authors declare that they have no competing interests.

\section{Author details}

${ }^{1}$ Neuro-Oncology Surgery Department of Fujian Cancer Hospital \& Fujian Medical University Cancer Hospital, Fuzhou 350014, China. ${ }^{2}$ Beijing Neurosurgical Institute, Capital Medical University, Beijing 100070, China. ${ }^{3}$ Department of Neurosurgery, Beijing Tiantan Hospital, Capital Medical University, Beijing 100070, China. ${ }^{4}$ Center of Brain Tumor, Beijing Institute for Brain Disorders, Beijing 100070, China. ${ }^{5}$ China National Clinical Research Center for Neurological Diseases, Beijing 100070, China.

Received: 30 June 2021 Accepted: 29 October 2021

Published online: 09 November 2021

\section{References}

1. Jiang T, et al. CGCG clinical practice guidelines for the management of adult diffuse gliomas. Cancer Lett. 2021;375:263-73. https://doi.org/10. 1016/j.canlet.2020.10.050.

2. Louis DN, et al. The $2021 \mathrm{WHO}$ classification of tumors of the central nervous system: a summary. Neuro-Oncology. 2020. https://doi.org/10.1093/ neuonc/noab106.

3. Jiang $\mathrm{T}$, et al. Clinical practice guidelines for the management of adult diffuse gliomas. Cancer Lett. 2020;499:60-72. https://doi.org/10.1016/j. canlet.2020.10.050.

4. Lin TK, et al. The long non-coding RNA LOC441204 enhances cell growth in human glioma. Sci Rep. 2017;7:5603. https://doi.org/10.1038/ s41598-017-05688-0.

5. Zhao CB, et al. MicroRNA-128-3p enhances the chemosensitivity of Temozolomide in glioblastoma by targeting c-met and EMT. Sci Rep. 2020;10:9471. https://doi.org/10.1038/s41598-020-65331-3.

6. Choi S, Yu Y, Grimmer MR, Wahl M, Chang SM, Costello JF. Temozolomideassociated hypermutation in gliomas. Neuro Oncol. 2018;20:1300-9. https://doi.org/10.1093/neuonc/noy016.

7. Wang J, et al. Clonal evolution of glioblastoma under therapy. Nat Genet. 2016;48:768-76. https://doi.org/10.1038/ng.3590.

8. Muscat AM, et al. The evolutionary pattern of mutations in glioblastoma reveals therapy-mediated selection. Oncotarget. 2018;9:7844-58. https:// doi.org/10.18632/oncotarget.23541.

9. Handing JW, Criss AK. The lipooligosaccharide-modifying enzyme LptA enhances gonococcal defence against human neutrophils. Cell Microbiol. 2015;17:910-21. https://doi.org/10.1111/cmi.12411.

10. Rose AM, Valdes RJ. Understanding the sodium pump and its relevance to disease. Clin Chem. 1994;40:1674-85.

11. Lefranc $F$, et al. Targeting the alpha 1 subunit of the sodium pump to combat glioblastoma cells. Neurosurgery. 2008;62:211-2. https://doi.org/ 10.1227/01.NEU.0000311080.43024.0E

12. Mijatovic T, et al. The alpha1 subunit of the sodium pump could represent a novel target to combat non-small cell lung cancers. J Pathol. 2007;212:170-9. https://doi.org/10.1002/path.2172.

13. Sakai $\mathrm{H}$, et al. Up-regulation of $\mathrm{Na}(+), \mathrm{K}(+)$-ATPase alpha 3-isoform and down-regulation of the alpha1-isoform in human colorectal cancer. FEBS Lett. 2004;563:151-4. https://doi.org/10.1016/S0014-5793(04)00292-3.

14. Mathieu V, et al. The sodium pump alpha1 sub-unit: a disease progression-related target for metastatic melanoma treatment. J Cell Mol Med. 2009;13:3960-72. https://doi.org/10.1111/j.1582-4934.2009.00708.x.

15. Shibuya $K$, et al. Increase in ouabain-sensitive K+-ATPase activity in hepatocellular carcinoma by overexpression of $\mathrm{Na}+, \mathrm{K}+-$ ATPase alpha 
3-isoform. Eur J Pharmacol. 2010;638:42-6. https://doi.org/10.1016/j. ejphar.2010.04.029.

16. Arrebola F, Zabiti S, Cañizares FJ, Cubero MA, Crespo PV, Fernández SE. Changes in intracellular sodium, chlorine, and potassium concentrations in staurosporine-induced apoptosis. J Cell Physiol. 2005;204:500-7. https://doi.org/10.1002/jcp.20306.

17. Tian J, et al. Changes in sodium pump expression dictate the effects of ouabain on cell growth. J Biol Chem. 2009;284:14921-9. https://doi.org/ 10.1074/jbc.M808355200

18. Minor NT, Sha Q, Nichols CG, Mercer RW. The gamma subunit of the Na,K-ATPase induces cation channel activity. Proc Natl Acad Sci USA. 1998;95:6521-5. https://doi.org/10.1073/pnas.95.11.6521.

19. Bao ZS, et al. RNA-seq of 272 gliomas revealed a novel, recurrent PTPRZ1-MET fusion transcript in secondary glioblastomas. Genome Res. 2014;24:1765-73. https://doi.org/10.1101/gr.165126.113.

20. Zhao Z, Meng FL, Wang W, Wang Z, Zhang CB, Jiang T. Comprehensive RNA-seq transcriptomic profiling in the malignant progression of gliomas. Sci Data. 2017;4:170024. https://doi.org/10.1038/sdata.2017.24.

21. Sweadner KJ, Wetzel RK, Arystarkhova E. Genomic organization of the human FXYD2 gene encoding the of the Na,K-ATPase. Biochem Biophys Res Commun. 2000;279:196-201. https://doi.org/10.1006/bbrc.2000.3907.

22. Béguin P, Crambert G, Guennoun S, Garty H, Horisberger JD, Geering K. CHIF, a member of the FXYD protein family, is a regulator of Na,K-ATPase distinct from the gamma-subunit. EMBO J. 2001;20:3993-4002. https:// doi.org/10.1093/emboj/20.15.3993.

23. Arystarkhova E, Donnet C, Asinovski NK, Sweadner KJ. Differential regulation of renal Na,K-ATPase by splice variants of the gamma subunit. J Biol Chem. 2002;277:10162-72. https://doi.org/10.1074/jbc.M111552200.

24. Hsu LL, et al. Targeting FXYD2 by cardiac glycosides potently blocks tumor growth in ovarian clear cell carcinoma. Oncotarget. 2016;7:6292538. https://doi.org/10.18632/oncotarget.7497.

25. Jin $\mathrm{M}$, et al. Expression mode and prognostic value of FXYD family members in colon cancer. Aging. 2021;13. https://doi.org/10.18632/aging. 203290.

26. Delprat B, Bibert S, Geering K. FXYD proteins: novel regulators of Na,KATPase. Med Sci (Paris). 2006;22:633-8. https://doi.org/10.1051/medsci/ 20062267633.

27. Wang $\mathrm{F}$, et al. FXYD2, a $y$ subunit of $\mathrm{Na}^{+}, \mathrm{K}^{+}$-ATPase, maintains persistent mechanical allodynia induced by inflammation. Cell Res. 2015;25:318-34. https://doi.org/10.1038/cr.2015.12.

\section{Publisher's Note}

Springer Nature remains neutral with regard to jurisdictional claims in published maps and institutional affiliations.
Ready to submit your research? Choose BMC and benefit from:

- fast, convenient online submission

- thorough peer review by experienced researchers in your field

- rapid publication on acceptance

- support for research data, including large and complex data types

- gold Open Access which fosters wider collaboration and increased citations

- maximum visibility for your research: over 100M website views per year

At BMC, research is always in progress.

Learn more biomedcentral.com/submissions 